\title{
Controlling the Antioxidant Activity of Green Tea Extract through Encapsulation in Chitosan-Citrate Nanogel
}

\author{
F. Piran, ${ }^{1}$ Z. Khoshkhoo $\mathbb{D}^{1},{ }^{1}$ S. E. Hosseini, ${ }^{2}$ and M. H. Azizi ${ }^{3}$ \\ ${ }^{1}$ Department of Food Science and Technology, North Tehran Branch, Islamic Azad University, Tehran, Iran \\ ${ }^{2}$ Department of Food Science and Technology, Tehran Science and Research Branch, Islamic Azad University, Tehran, Iran \\ ${ }^{3}$ Department of Food Science and Technology, Faculty of Agriculture, Tarbiat Modares University, Tehran, Iran
}

Correspondence should be addressed to Z. Khoshkhoo; zh_khoshkhoo@iau-tnb.ac.ir

Received 16 December 2019; Revised 24 February 2020; Accepted 26 March 2020; Published 3 July 2020

Academic Editor: Anet Režek Jambrak

Copyright $(2020$ F. Piran et al. This is an open access article distributed under the Creative Commons Attribution License, which permits unrestricted use, distribution, and reproduction in any medium, provided the original work is properly cited.

\begin{abstract}
Applying bioactive ingredients in the formulation of foods instead of artificial preservatives is problematic because bioactive ingredients are unstable and sensitive to environmental conditions. The present study aimed to control the antioxidant activity of green tea extract (GT) through encapsulating in chitosan nanoparticles (CS-NP). The synthesized nanoparticles were analyzed by using scanning electron microscopy (SEM), Fourier transform infrared spectroscopy (FT-IR), and X-ray diffraction (XRD). The encapsulation efficiency (EE), particle size, zeta potential, and polydispersity index (PDI) of GT-loaded CS-nanoparticles (CS-NPGT) were assessed. Based on the results, the particle size and zeta potential related to the ratio of CS to GT of $1: 0.5$ were obtained as $135.43 \pm 2.52 \mathrm{~nm}$ and $40.40 \pm 0.2 \mathrm{mV}$, respectively. Furthermore, the results of FT-IR and XRD confirmed the validity of encapsulating GT in CS-NP. In addition, the antioxidant activity of GT increased after nanoencapsulation since the $\mathrm{IC}_{50}$ value of CS-NP-GT decreased to $6.13 \pm 0.12 \mu \mathrm{g} / \mathrm{ml}$. Finally, applying these particles for delivering GT polyphenols in foods is regarded as promising.
\end{abstract}

\section{Introduction}

During the past few decades, the trend of using natural antioxidants and plant-derived extracts instead of chemical preservatives and synthetic antioxidants for extending the shelf life of food products has received increased attention [1]. The green tea extract derived from Camellia sinensis L. leaf is rich in bioactive compounds (polyphenols) with high antioxidant activity and healthy properties [2]. In a number of studies, green tea extract showed comparable antioxidant performance to common synthetic antioxidant tert-butylhydroquinone (TBHQ) and may be more effective than other natural sources of antioxidants [3]. Green tea polyphenols mainly include $(+)$-catechins, (-)-epicatechins, (+)-gallocatechins, (-)-epicatechin gallate, (-)-epigallocatechin, and (-)-epigallocatechin gallate [4]. Catechins neutralize free radicals and active oxygen species by donating electrons due to the existence of aromatic ring and hydroxyl groups in their structure [3]. However, using catechins as natural antioxidants in food industry is limited due to chemical instability and high sensitivity to factors such as temperature, light, oxygen, enzymes, alkaline $\mathrm{pH}$, and change in the aroma and flavour of food materials [5]. Therefore, to overcome these limitations, various new technologies such as nanoencapsulation have been developed. The chitosan nanoparticles (CS-NP) having a controlled shape and size can carry sensitive compounds, such as catechins, and protect them against destructive factors in food systems through encapsulation [6]. Chitosan (CS) polymer with excellent properties such as biodegradation, biocompatibility, cationic charge, and intrinsic antimicrobial potential is used as a carrier and wall to create and develop nanogels systems [7]. Nanogels are cross-linked three-dimensional polymer chain networks, which are created through covalent linkages. Cross-linking methods are different, such as ionic cross-linking, cross-linking polymerization, radiation cross-linking, and functional group cross-linking [8]. 
Natural polysaccharides, such as chitosan in order to be used to produce nanogels, could be modified by using hydrophilic moieties like citrate. No study, to the best of our knowledge, has been conducted on nanoencapsulating green tea (GT) by citrate polyanion and chitosan polymer. The extract of GT was used in the present study since using pure catechin in food industry is noneconomic, and the purification process is time- and cost-consuming. The present study sought to synthesize chitosan-citrate nanogel through forming a bond between the amino group of chitosan $((1,4)$ 2-amino-2-desoxy- beta-D-glucan) and carboxyl group of citrate (2-hydroxypropane-1,2,3-tricarboxylic acid). The final CS-citrate nanogel is formed by aggregation of chitosan nanoparticles. After creating the nanogel, GT was loaded in it. Further, the physicochemical properties and antioxidant activity of GT and GT-loaded CS nanoparticles (CS-NP-GT) were assessed.

\section{Materials and Methods}

2.1. Extracting GT. Fresh GT was prepared from C. sinensis leaves from the tea gardens of Lahijan in Iran. Unwashed GT leaves were dried for five days at ambient temperature under natural airflow. The dried samples were crushed by a mill and obtained powder was kept at $-18^{\circ} \mathrm{C}$ until extracting GT. In order to prepare GT aqueous extract, 10 gr of dried tea leaf powder was added into $150 \mathrm{ml}$ of distilled water with $80^{\circ} \mathrm{C}$ and extracted for $7 \mathrm{~min}$ at the fixed temperature of $80^{\circ} \mathrm{C}$. Then, it was centrifuged to separate impurities, filtered by using filter paper, dried completely through freeze-drying method (Zirbus, Germany) and kept in coloured dishes at refrigerator temperature for further steps.

\subsection{Encapsulating GT in $C S-N P$}

2.2.1. Materials. Chitosan (75-85\% deacetylation, low molecular weight), N-hydroxysuccinimide (NHS), and 1ethyl-3-(3-dimethylaminopropyl) carbodiimide (EDC) were provided by Sigma-Aldrich Chemical Co. Furthermore, citric acid $\left(\mathrm{C}_{6} \mathrm{H}_{8} \mathrm{O}_{7}\right)$, hydrochloric acid $(\mathrm{HCl})$, and sodium hydroxide $(\mathrm{NaOH})$ were provided by Merck, Germany. Ultrasonic homogenizer $(400 \mathrm{~W}$; Hielscher ultrasonic, Germany) was used in the present study.

\subsubsection{Synthesizing Chitosan-Citrate Nanogel. CS-NP was} provided through Darras et al.'s [9] method with some modifications. To this end, 1 gr of chitosan powder was added into the distilled water containing $2 \mathrm{ml}$ of hydrochloric acid and dissolved using a magnetic stirrer at $250 \mathrm{rpm}$ to make the final chitosan concentration of $1 \%$ and sonicated for $20 \mathrm{~min}$ at room temperature by using ultrasonic homogenizer ( $400 \mathrm{~W}$; Hielscher ultrasonic, Germany) with $60 \%$ of maximum power. Further, the solution containing $0.1 \mathrm{gr}$ of citric acid dissolved in distilled water, $0.1 \mathrm{gr}$ of EDC, and $0.1 \mathrm{gr}$ of NHS was added at room temperature to the chitosan solution stirring at $250 \mathrm{rpm}$ and stirred for $2 \mathrm{~h}$. Covalent bonds were formed in this step between $\mathrm{NH}_{3}{ }^{+}$ groups with positive charge and $\mathrm{COO}^{-}$groups of citrate.
Furthermore, $\mathrm{pH}$ of CS-citrate solution was set at around 7 by using diluted soda to precipitate chitosan-citrate. The precipitate was separated by centrifuging at $9000 \mathrm{rpm}$ for $18 \mathrm{~min}$ and washed by ethanol three times and distilled water one time to remove impurities. chitosan-citrate nanogel became soluble in the next step by setting $\mathrm{pH}$ at 4 and was sonicated for $10 \mathrm{~min}$ by using ultrasonic homogenizer with $60 \%$ of maximum power.

2.2.3. Loading GT in CS-NP. Controlling antioxidant properties of GT through encapsulating in chitosan-citrate nanogel was regarded as the main objective of the present study. Regarding loading GT with $20000 \mu \mathrm{g} / \mathrm{ml}$ in CS-NP, 0.1 gr of dried GT was dissolved in $5 \mathrm{ml}$ of distilled water and added slowly to the CS-citrate nanogel sonicating at $80 \mathrm{~Hz}$, and then sonication continued for $7 \mathrm{~min}$. The absorption of GT was assessed through the process of emitting into chitosan-citrate nanoparticles due to a high concentration of chitosan-citrate nanoparticles, which can absorb the great amount of water.

2.3. Characterization of Nanoparticles. The particle size, PDI, and zeta potential of CS-NP-GT were measured using the Zetasizer Nano ZS (Malvern Instruments Ltd., Malvern, UK) equipped with $\mathrm{He}-\mathrm{Ne}$ laser at the wavelength of $633 \mathrm{~nm}$, fixed scattering angle of $90^{\circ}$ and power of $4 \mathrm{mV}$.

The morphological properties of CS-NP and CS-NP-GT (with CS : GT ratio of $1: 0.5$ ) were evaluated using the images related to scanning electron microscopy (SEM) (FEI ESEM Quanta 200, USA). In this regard, $1 \mathrm{ml}$ of CS-NP or CS-NPGT was diluted by $5 \mathrm{ml}$ of double distilled water and placed in an ultrasonic bath (Elma, Germany) at $60 \mathrm{~Hz}$ for $5 \mathrm{~min}$. Further, $1 \mathrm{ml}$ of intended solution was placed on a clean glass slide and dried at room temperature. Furthermore, slide was coated by a gold layer by using coater (CoXEM, South Korea) for the surface conductivity of sample and prepared for observing with SEM.

Regarding the determination of the chemical structure of CS-NP and CS-NP-GT, the samples were completely dried through freeze-drying technique (Zirbus, Germany), and their FTIR spectra were assessed using FTIR spectrometer (Thermo Scientific Nicolet, USA) in the range of $400-4000 \mathrm{~cm}^{-1}$ with the resolution of $4 \mathrm{~m}^{-1}$. Further, the FTIR spectra of chitosan and GT were applied to obtain the structure of primary materials.

In order to assess the X-ray diffraction pattern (XRD) of CS, CS-NP, CS-NP-GT, and GT, CS-NP and CS-NP-GT were freeze-dried, and X'Pert MPD (Philips, The Netherlands) was used. $\mathrm{Cu}-\mathrm{K} \alpha \mathrm{X}$-ray source was set at $30 \mathrm{~mA}$ and $40 \mathrm{kV}$, rays were collected in the angle range of $2 \theta=5-50$, and the diagram of their reflection was plotted.

2.4. Determining Encapsulation Efficiency. Encapsulation efficiency (EE) was measured based on the method provided by Rafiee et al. [10]. To this end, the suspension of CS-NPGT was prepared and centrifuged at $30000 \mathrm{rpm}$ for $1 \mathrm{~h}$ at $4^{\circ} \mathrm{C}$ (3-30 k, Sigma, Germany) to separate the incorporated 
phenolic compounds from free ones. Further, Folin-Ciocalteu method was used to measure the amounts of total phenolic compounds in initial suspension and the supernatant. The percentage of EE was determined through the following equation:

$$
\mathrm{EE} \%=\left(\frac{C_{\text {initial }}-C_{\text {sup }}}{C_{\text {initial }}}\right) \times 100,
$$

where $C_{\text {initial }}$ and $C_{\text {sup }}$ are the initial and unloaded phenolic compounds determined in the supernatant, respectively.

2.5. Evaluation of Antioxidant Activity. The antioxidant activity was evaluated by scavenging radical activity using the 2,2-diphenyl-2 picrylhydrazyl (DPPH) radical method, which was described by Afolayan et al. with some modifications [11]. To this end, a solution of $0.135 \mathrm{mM}$ DPPH in methanol $(0.0053 \mathrm{gr}$ in $100 \mathrm{ml})$ was prepared. GT and CSNP-GT were dissolved in water to obtain final concentrations of $5-333 \mu \mathrm{g} / \mathrm{ml}$. CS-NP-GT samples were stirred for four hours at $200 \mathrm{rpm}$ to release GT from CS-NP. Further, $1.5 \mathrm{ml}$ of the samples solutions was poured into $1.5 \mathrm{ml}$ of DPPH solution in test tube. Control solution was prepared by using $1.5 \mathrm{ml}$ of methanol instead of extract. Ascorbic acid (in concentration $1,2,4,8$, and $15 \mu \mathrm{g} / \mathrm{ml}$ ), as a standard substance, was added to DPPH solution using the previously mentioned method. The experiment was repeated three times in each concentration. After placing the solutions in a dark environment for $30 \mathrm{~min}$, the CS-NP-GT samples were ultracentrifuged for $3 \mathrm{~min}$ to achieve clear solutions. The absorbance of samples was measured by using UV-spectrophotometer (Human crop, Xma-2000) at $517 \mathrm{~nm}$. The percentage of radical scavenging activity (RSA) was determined by using the following equation:

$$
\operatorname{RSA}(\%)=\left[\frac{1-(S-S B)}{C}\right] \times 100,
$$

where $\mathrm{S}, \mathrm{SB}$, and $\mathrm{C}$ are, respectively, the absorbance of the sample (DPPH + extract) and blank (methanol + extract), and control $(\mathrm{DPPH}+$ methanol). After determining RSA percentage, the $\mathrm{IC}_{50}$ of GT, CS-NP-GT, and ascorbic acid were obtained by plotting the amounts of RSA versus the different concentrations of sample and calculating regression line equation [12].

2.6. Determining the Total Phenolic Content (TPC). TPC of GT and CS-NP-GT was evaluated using Folin-Ciocalteu reagent. In this regard, the different concentrations of the standard solutions of gallic acid in water and GT were prepared $(\mu \mathrm{gr} / \mathrm{ml})$. Further, $1 \mathrm{ml}$ of prepared samples and standard solutions was added into $10 \mathrm{ml}$ flasks containing $5 \mathrm{ml}$ of distilled water. $0.5 \mathrm{ml}$ of Folin-Ciocalteu reagent was added to each flask, samples and standard solutions flask individually, stirring the solutions well. After $3 \mathrm{~min}, 1 \mathrm{ml}$ of $20 \%$ sodium carbonate solution, which was previously prepared, was poured into $10 \mathrm{ml}$ reaction flasks and made to volume with distilled water immediately. Finally, the absorbance of all solutions was measured after $1 \mathrm{~h}$ by using
UV-spectrophotometer at $725 \mathrm{~nm}$. Blank solution included $5 \mathrm{ml}$ of water, $0.5 \mathrm{ml}$ of Folin-Ciocalteu reagent, and $1 \mathrm{ml}$ of $20 \%$ saturated sodium carbonate, which was made up to volume. Additionally, TPC was reported as mgr gallic acid equivalent (GAE/gram) [13]. TPC of CS-NP-GT was determined through this method except that it was first ultracentrifuged for $3 \mathrm{~min}$ and then its absorbance was measured.

\section{Results and Discussion}

3.1. Size, Zeta Potential, PDI, and Morphology of Nanoparticles. The effects of the different concentrations of GT on particle size, PDI, zeta potential, and encapsulation efficiency are summarized in Table 1. Particle size, zeta potential, and PDI were measured using Dynamic Light Scattering (DLS) technique.

As shown in Table 1, the particle size increases significantly by loading GT in CS-NP $(p<0.05)$, while a significant reduction is observed in zeta potential $(p<0.05)$. Decreasing zeta potential which occurs by loading more GT in CS-NP may be related to the reduction of free amine groups in the surface of CS-NP due to their interaction with GT. This decreasing trend of zeta potential and increasing particle size were reported after loading clove essence [14], ellagic acid [15], and carvacrol [11] in nanoparticles. Based on the results in Table 1, the particle size increased by adding the ratio of GT to CS. Particle size could be related to the particle size of GT since chitosan-citrate nanoparticles were synthesized at first and then GT was loaded on it.

The size of CS-NP particles (CS:GT=1:0) was small because of sonicating CS solution for $20 \mathrm{~min}$ in the first step of synthesizing CS-NP. The cavitation energy of ultrasound waves can cleave the $\beta$-1,4-glucosidic bonds of chitosan which resulted in reducing the particle size without any change in the deacetylation degree of chitosan and zeta potential of particles [16].

Table 1 represents the values of the zeta potential related to all concentrations of CS: GT, indicating that all values are positive due to the presence of the amine groups of chitosan on the surface of nanoparticles. The positive charge of CSNP prevents particles from adhering to each other due to repulsive force. Thus, the particles remain suspended and fail to coagulate and precipitate. Adding the cross-linker having carboxyl groups with negative charge leads to reduction of zeta potential, while the particles adhere to each other and precipitate by pouring more values.

PDI range was obtained as $0.113 \pm 0.005-0.323 \pm 0.021$. PDI is regarded as an index of the homogeneity of nanoparticles, where less values represent more homogeneity. Further, the particle size of nanoparticles becomes heterogeneous by adding PDI to more than 0.3. The PDI of nanoparticles increases over the time because of adherence of nanoparticles and reducing the homogeneity [17]. PDI is affected by some factors, such as solution temperature, reaction time, concentration, molecular weight of chitosan, and method used for stirring [18]. The shape and morphology of CS-NP-GT particles were observed by scanning electron microscopy. As shown in Figure 1, CS-NP-GT is 
TABLE 1: Particle size, zeta potential, PDI, and encapsulation efficiency (EE) of CS-NP and CS-NP-GT prepared with different ratios of CS: GT.

\begin{tabular}{lcccc}
\hline CS : GT ratio & Particle size $(\mathrm{nm})$ & Zeta-potential $(\mathrm{mV})$ & PDI & Encapsulation efficiency \\
\hline $1: 0$ & $112.74 \pm 1.56^{\mathrm{d}}$ & $41.83 \pm 0.1^{\mathrm{a}}$ & $0.113 \pm 0.005^{\mathrm{d}}$ & $0^{\mathrm{c}}$ \\
$1: 0.25$ & $125.38 \pm 1.57^{\mathrm{c}}$ & $40.83 \pm 0.1^{\mathrm{b}}$ & $0.323 \pm 0.021^{\mathrm{a}}$ & $48.93 \pm 0.26^{\mathrm{b}}$ \\
$1: 0.5$ & $135.43 \pm 2.52^{\mathrm{c}}$ & $40.40 \pm 0.2^{\mathrm{c}}$ & $0.296 \pm 0.005^{\mathrm{b}}$ & $68.37 \pm 1.17^{\mathrm{a}}$ \\
$1: 0.75$ & $243.28 \pm 2.13^{\mathrm{b}}$ & $38.75 \pm 0.1^{\mathrm{d}}$ & $0.300 \pm 0.100^{\mathrm{b}}$ & $65.92 \pm 2.67^{\mathrm{a}}$ \\
$1: 1$ & $375.06 \pm 3.10^{\mathrm{a}}$ & $35.69 \pm 0.4^{\mathrm{e}}$ & $0.233 \pm 0.005^{\mathrm{c}}$ & $67.01 \pm 1.67^{\mathrm{a}}$ \\
\hline
\end{tabular}

Results were reported as mean $\pm \mathrm{SD}(n=3)$. Different letters in the same column indicate significant differences among means (LSD test, $p<0.05)$. PDI: polydispersity index; CS-NP: chitosan nanoparticle; CS-NP-GT: green tea extract-loaded chitosan nanoparticle.
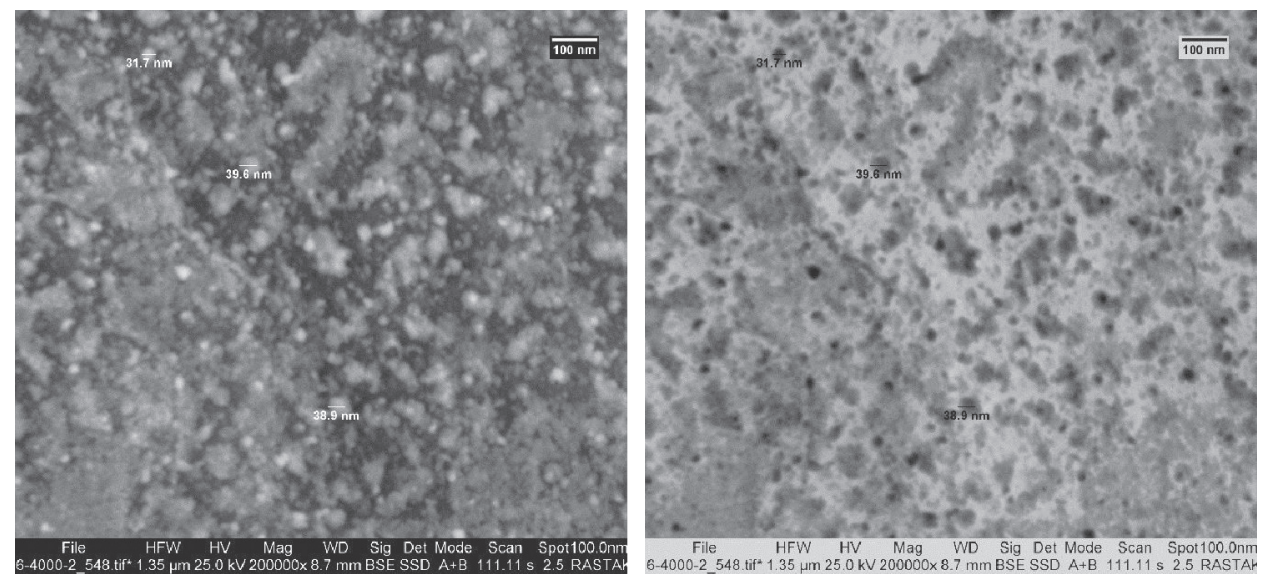

(a)
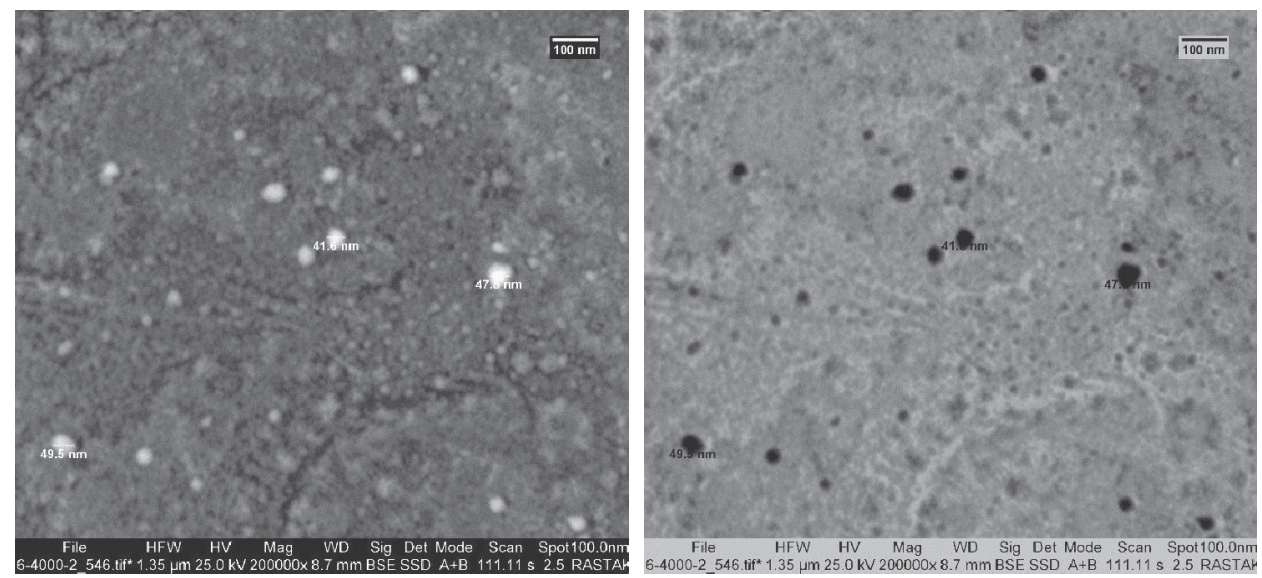

(b)

FIGURE 1: Scanning electron microscopy (SEM) micrographs of CS-NP (a) and GT-CS-NP (b) prepared using an initial weight ratio of CS : GT of $1: 0.5$.

observed in both forms of nanoparticles, single and adhering.

These particles are agglomerated and adhered in most parts due to their adhesion during preparing and drying the sample for observing with SEM. Furthermore, the particles' size in the SEM image are less than $100 \mathrm{~nm}$ and their mean size is less than that is measured by DLS method $(135.43 \mathrm{~nm}, \mathrm{CS}: \mathrm{GT}=1: 0.5)$ due to some reasons including the sample being completely dried in the step of preparing CS-NP-GT for SEM, while nanoparticles dispersed in aqueous environment were swollen and hydrated by absorbing water, leading to an increase in their size when measured by the DLS method. Woranuch and Yoksan [11] reported that the particle size obtained by DLS method (586-683 nm) was larger compared to that observed in TEM image $(80-100 \mathrm{~nm})$ because of hydrating in DLS method. Similar results were reported by LuqueAlcaraz et al., [19] as the particle size measured by SEM was smaller compared with that measured by DLS method.

Figure 2 shows the size and zeta potential distribution intensity graphs of CS-NP and CS-NP-GT prepared with different ratios of CS:GT. These graphs belong to the size and zeta values in Table 1, measured by the DLS method. 

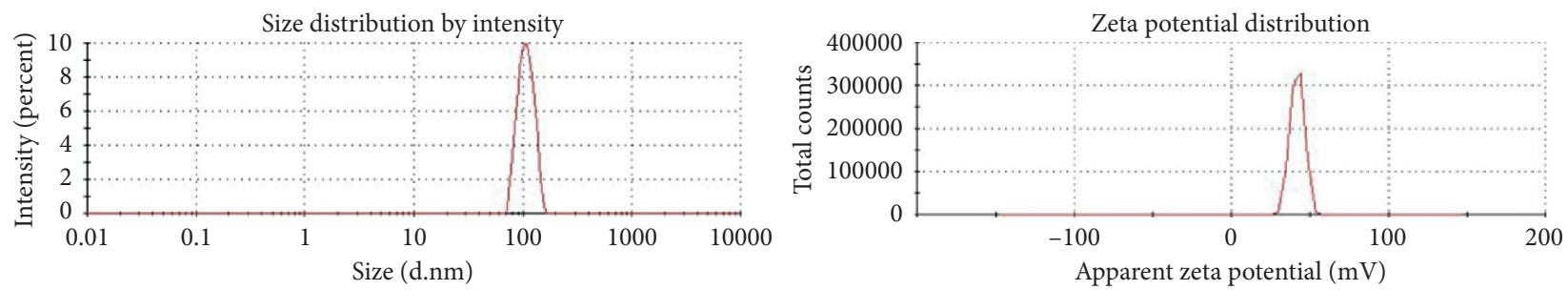

(a)
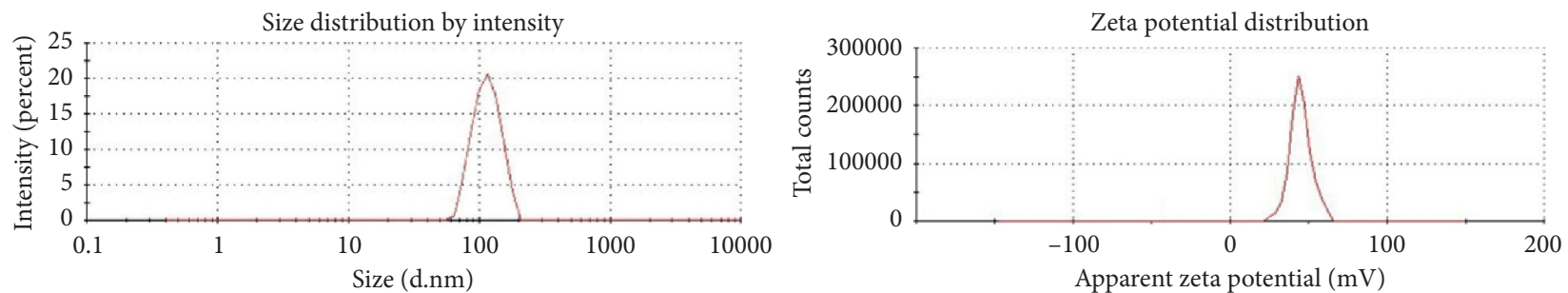

(b)
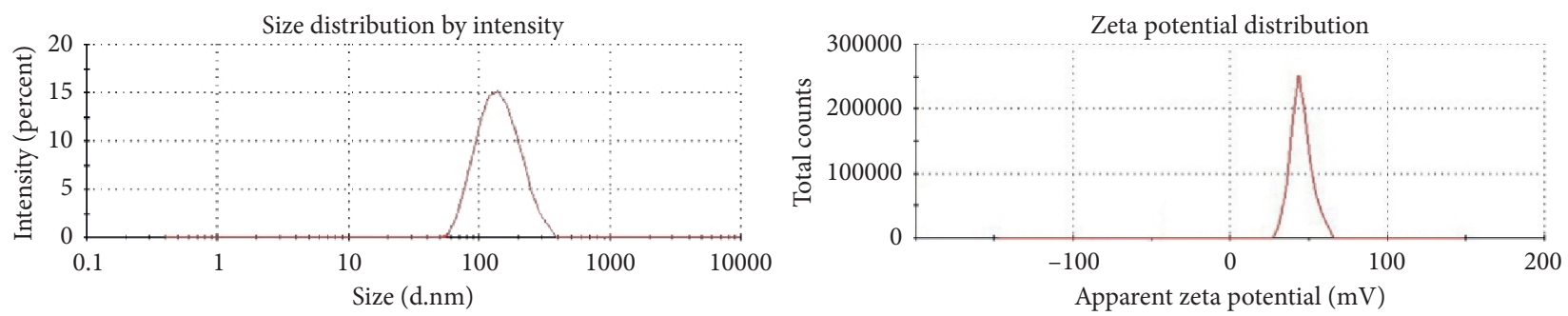

(c)
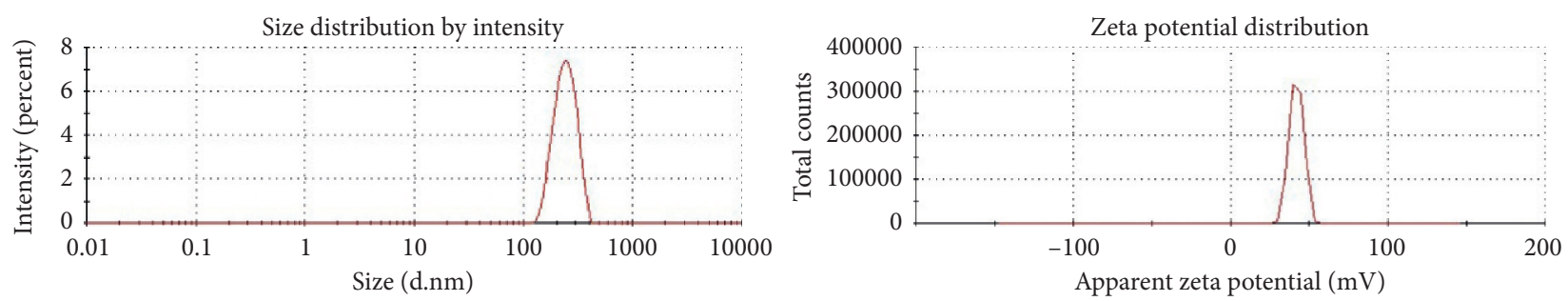

(d)
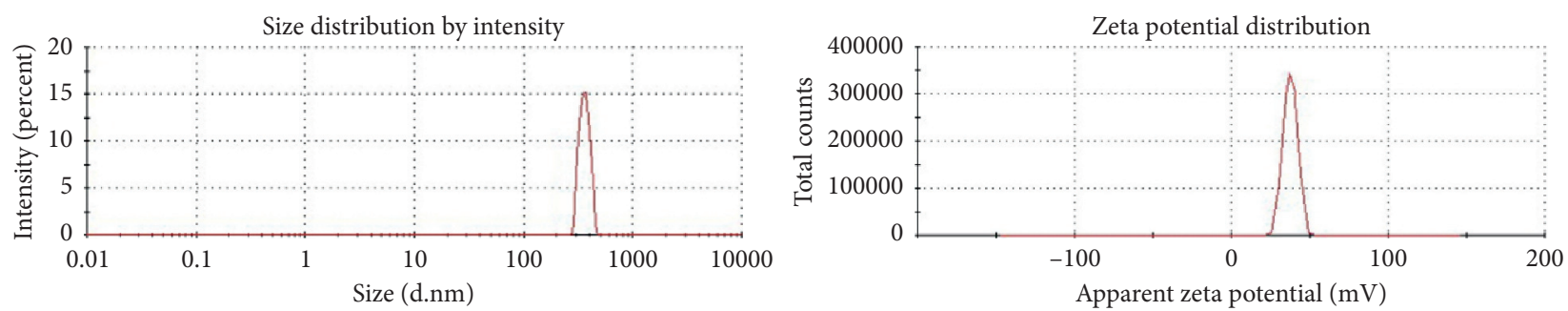

(e)

FIGURE 2: Size distribution intensity and zeta potential distribution intensity graphs of CS-NP and CS-NP-GT synthesized with different ratios of CS: GT: (a) CS:GT=1:0; (b) CS: GT =1:0.25; (c) CS:GT=1:0.5; (d) CS:GT=1:0.75; (e) CS:GT =1:1.

3.2. FT-IR Analysis. The FT-IR spectra of neat CS and CS$\mathrm{NP}$ are presented in Figure 3. As shown, neat CS spectrum exhibits main characteristic absorbencies of carbonyl $(\mathrm{C}=\mathrm{O})$ and amine $\left(-\mathrm{NH}_{2}\right)$ groups at $1659 \mathrm{~cm}^{-1}$ and $1565 \mathrm{~cm}^{-1}$, respectively. Asymmetric and symmetric stretching of $\mathrm{C}=\mathrm{O}$ at $1659 \mathrm{~cm}^{-1}$ and around $1400 \mathrm{~cm}^{-1}$ are related to the presence of residual amide I in the CS backbone which is originated from chitin structure.
In addition, primary amines present a critical absorbency between 3500 and $3400 \mathrm{~cm}^{-1}$, which could be ascribed to the asymmetric and symmetric stretching of $\mathrm{N}-\mathrm{H}$ links. On the other hand, the strong absorption creates a broad peak due to the stretching vibration of $-\mathrm{NH}_{2}$ overlapped onto the $-\mathrm{OH}$ group absorbency, which could be detected at $3400-3500 \mathrm{~cm}^{-1}$. A robust peak can be observed at $2879 \mathrm{~cm}^{-1}$, which is related to the stretching 


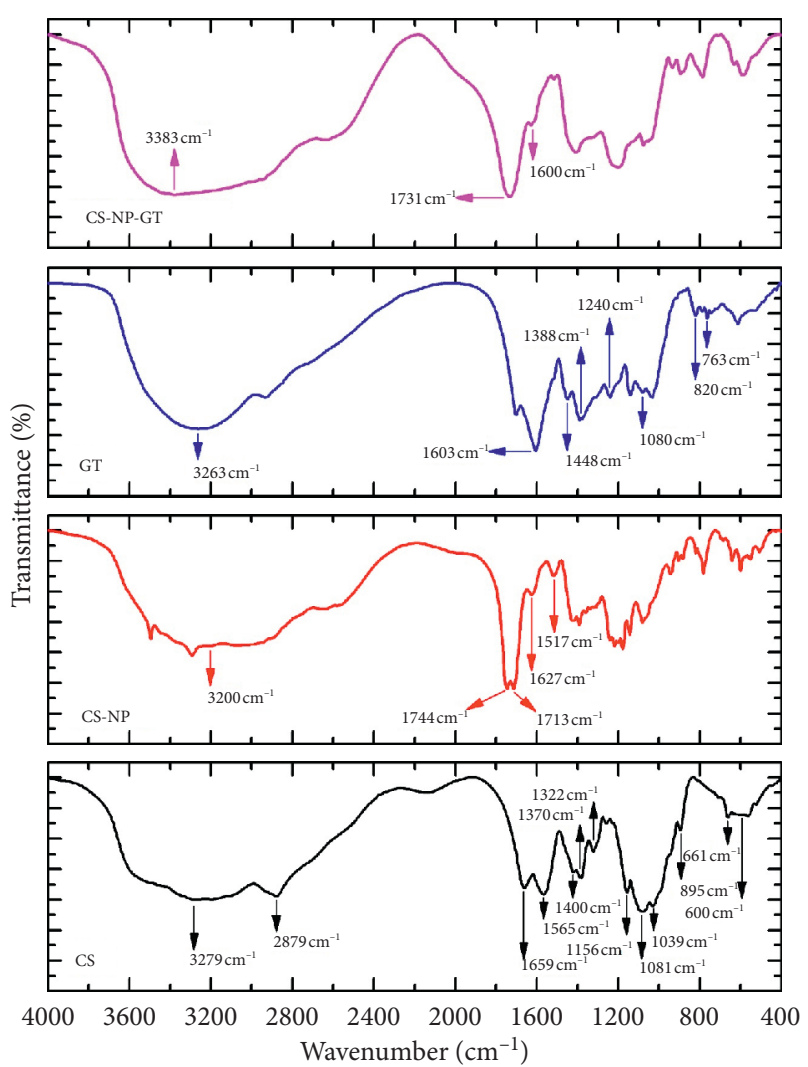

FIGURE 3: FT-IR spectra of CS-NP-GT (CS : GT = 1: 0.5), GT, CS$\mathrm{NP}$, and CS.

vibration of allylic $\mathrm{C}-\mathrm{H}$ groups in the $\mathrm{CS}$ ring. The absorbency peaks in the range of $1000-1200 \mathrm{~cm}^{-1}$ are ascribed to the saccharide structure of CS backbone. The absorbencies at 1380,1322 , and $1156 \mathrm{~cm}^{-1}$ were originated from the $\mathrm{C}-\mathrm{O}$ stretching, $\mathrm{OH}$ in plane bending and $\mathrm{C}-\mathrm{O}-\mathrm{C}$ bonds (the glycosidic linkage between CS units), respectively. The peaks at 1070 and $1030 \mathrm{~cm}^{-1}$ are related to $\mathrm{C}-\mathrm{OH}$ stretching and $\mathrm{C}-\mathrm{N}$ vibrations, and absorbency at $895 \mathrm{~cm}^{-1}$ is related to $\mathrm{C}-\mathrm{C}$ stretching vibration. The peak at $661 \mathrm{~cm}^{-1}$ reveals of $\mathrm{N}-\mathrm{H}$ wagging vibration. Furthermore, the bending vibration in the CS ring occurs at about $600 \mathrm{~cm}^{-1}$ [20]. In the polymerization media, CS backbone cross-linked with citrate molecules, as the citrate with the help of EDC and NHS could produce cross-links via the interaction between its carboxylic parts with amino groups of CS (Figure 4) [21].

Comparing the FT-IR spectra of CS with CS-NP indicated that CS backbone was covalently cross-linked with citrate through reaction between $-\mathrm{NH}_{2}$ in $\mathrm{CS}$ and $-\mathrm{COOH}$ in citrate. In this way, the intensity of absorbency at about $1565 \mathrm{~cm}^{-1}$, related to the $-\mathrm{NH} 2$ bending vibration in the CS backbone, significantly reduced in the FT-IR spectrum of CS-NP. Furthermore, the wave numbers of $-\mathrm{NH}_{2}$ bending vibration and $\mathrm{C}=\mathrm{O}$ stretching of CS backbone noticeably shifted from 1565 to $1659 \mathrm{~cm}^{-1}$ and from 1517 to $1627 \mathrm{~cm}^{-1}$, respectively. In addition, these observations also confirm the possibility of a chemical interaction (cross-links) between citrate and CS. The robust absorbencies with dual peak nature at $1713-1744 \mathrm{~cm}^{-1}$ are related to the stretching vibration of the carbonyl group remaining from citrate, which was useful for the adsorbing polar molecules in the encapsulation process.

Based on the spectrum of GT in Figure 3, the significant absorbency at $1606 \mathrm{~cm}^{-1}$ due to $\mathrm{C}=\mathrm{C}$ stretching corresponds to the aromatic ring of phenolic structures. In addition, the bending absorbency of $\mathrm{C}-\mathrm{C}$ in aromatic ring occurred at $1448 \mathrm{~cm}^{-1}$. OH deformation of the aromatic alcohol appeared at 1388 and $1240 \mathrm{~cm}^{-1}$. At about $1080 \mathrm{~cm}^{-1}$, another band is observed due to an aromatic ring stretch. The absorbency peaks due to the aromatic $\mathrm{OH}$ wagging are observed between 850 and $750 \mathrm{~cm}^{-1}$. Further, a broad peak due to the $\mathrm{OH}$ stretch is observed at around $3263 \mathrm{~cm}^{-1}$. Compared with CS-NP spectrum occurrence, the peak around $1600 \mathrm{~cm}^{-1}$ due to aromatic $\mathrm{C}=\mathrm{C}$ stretching is a sign of green tea extract in the nanoparticles. Finally, the variation in intensity and amplitude of the peak at around $3400 \mathrm{~cm}^{-1}$ due to the hydroxyl groups in the sample loaded by GT extract indicates the hydrogen bonding interactions between the hydroxyl groups of CS with the polyphenolic groups in GT. The previously mentioned observation confirms that the GT is encapsulated in the nanoparticles after cross-linking CS. These results are in good agreement with those reported previously by Siripatrawan and Harte [22] who studied the physicochemical interactions between CS and catechins. They found that the amino and hydroxyl groups of the chitosan decreased when incorporated with GT catechins. Furthermore, the same results were found by Curcio et al. [23] regarding the formation of covalent insertion of antioxidant molecules like gallic acid and catechin on CS.

3.3. XRD Analysis. Figure 5 displays the XRD patterns of CSNP-GT, GT, CS-NP, and CS.

The results indicated that the reflection around $2 \theta=11.95^{\circ}$ manifests the presence of the crystal form I and the intense reflection at $2 \theta=23.4^{\circ}$ is related to the crystal form II [24]. It is worth noting that CS always surrounds bound water (5\%) even if it is considerably dried. The crystalline peak centered at around $2 \theta=11.95^{\circ}$ (peak I) is ascribed to the hydrated crystalline structure of CS. Another peak observed near $2 \theta=23.4^{\circ}$ (peak II) is considered as the clue of the moderately regular crystal lattice in chitosan [25]. A characteristic peak at around $2 \theta=23.4^{\circ}$ indicates the high degree of crystallinity in neat CS. As shown in the XRD pattern of CS-NP, the peak at $2 \theta=11.95^{\circ}$ was eliminated, and the characteristic peak at $2 \theta=23.3^{\circ}$ is broaden. Finally, the intensity of the latter reduced significantly. Based on the previously mentioned observations, an amorphous nature of the CS chains was reported in the nanoparticles. The crosslinker (citrate molecules) diffused properly between the CS chains which had already been dissolved in the reaction media. Therefore, the occurrence of the cross-linking reaction prevents rearranging CS chains into a dense crystal structure. The neat extract exhibited one characteristic broadened peak at $2 \theta=24.11^{\circ}$ indicating the amorphous 


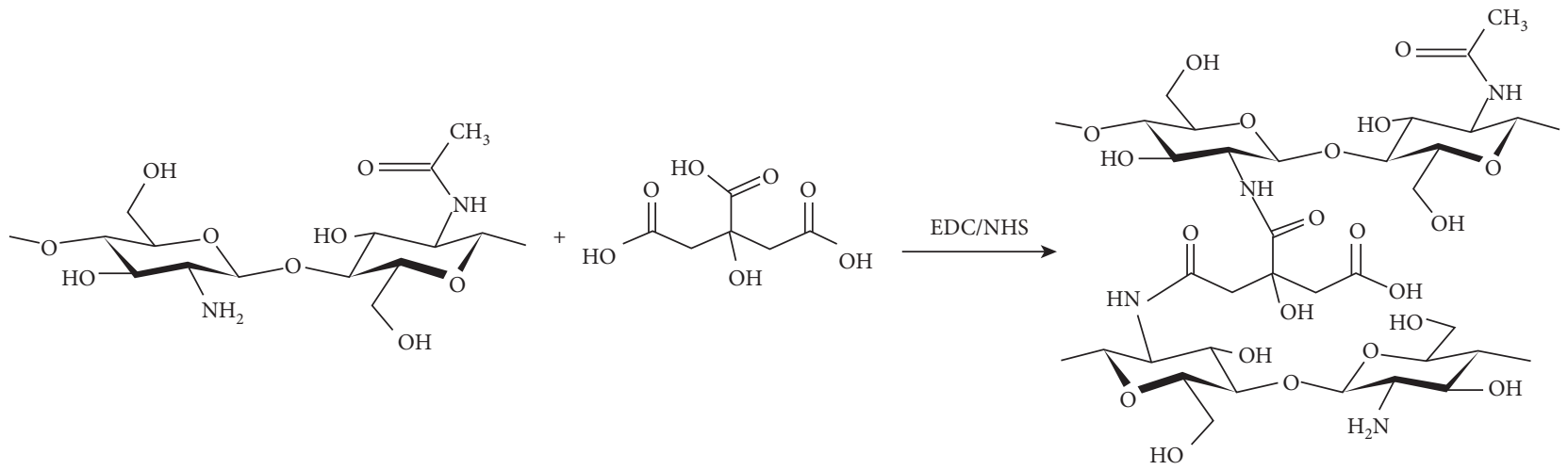

FIGURE 4: Synthesis of CS-citrate nanogel (structure in the right) from CS and citrate using EDC and NHS as the activation agents.

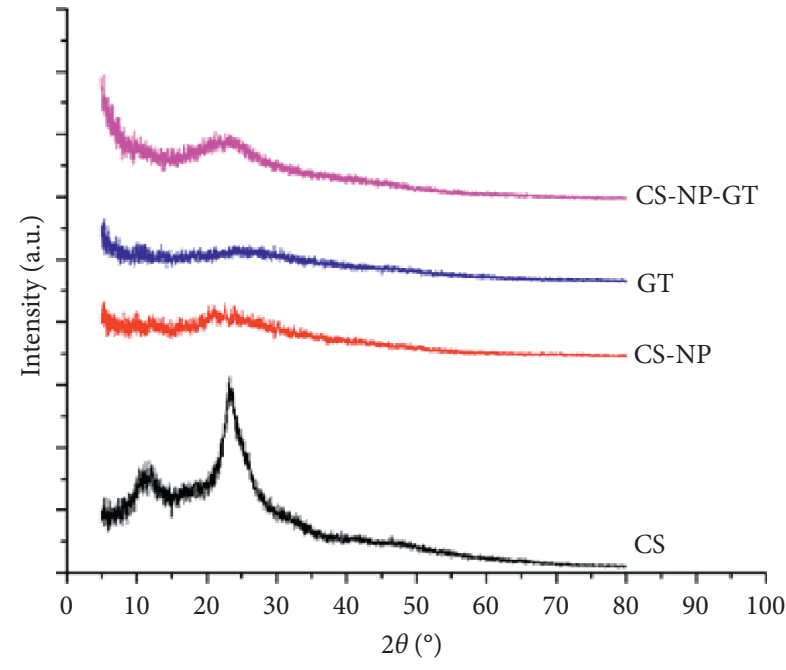

Figure 5: XRD of single ingredients and their complex samples of CS-NP-GT (CS: GT = $1: 0.5$ ), GT, CS-NP, and CS.

nature of the extract. Regarding the comparison of the patterns, no significant difference was found between the XRD patterns of the CS-NP and CS-NP-GT. The latter confirmed the amorphous structure of nanoparticles after loading process and the presence of GT within chitosan nanoparticles. In this regard, the characteristics peaks of GT at $2 \theta=24.11^{\circ}$ and CS-NP-GT at $2 \theta=23.23^{\circ}$ were merged into each other and just a broadened peak appeared around $2 \theta \sim 23^{\circ}$. The results are inconsistent with those reported previously by Liang et al. [26], who studied the encapsulation of epigallocatechin gallate in nanoparticles. They found that epigallocatechin gallate encapsulated with chitosan nanoparticles showed flatter peak rather than sharp peaks. The epigallocatechin-gallate-loaded CS nanoparticles did not exist in a crystalline form, which results in providing additional evidence of encapsulation.

3.4. Encapsulation Efficiency. As shown in Table 1, the maximum percentage of EE is obtained in the CS : GT ratio of $1: 0.5$ as $68.37 \pm 1.17 \%$. Adding the concentration of GT and phenolic compounds versus CS-NP from 0 to 0.5 leads to an increase in the $\mathrm{EE} \%$, while this percentage becomes fixed by enhancing GT concentration more than 0.5 and no significant difference is observed between $\mathrm{EE} \%$ (using similar letters representing the lack of significant difference). Gan and Wang [27] encapsulated bovine serum albumin (BSA) in CS-NP and found that $\mathrm{EE} \%$ increased by decreasing CS concentration and adding serum concentration due to viscosity reduction.

3.5. Antioxidant Activity. DPPH method is regarded as a fast and extensive approach to assess antioxidant activity and power of combining with free radicals or donating hydrogen in food materials. DPPH radical (2,2-diphenyl-1-picrylhydrazyl) is a stable free radical with the central atom of nitrogen. DPPH changes from purple to yellow with reduction reaction through capturing hydrogen or electron [28]. Figure 6 displays the scavenging activity (\%) of CS-NP-GT, GT, and ascorbic acid versus concentration by representing high antioxidant activity of GT and CS-NP-GT. The range of GT and CS-NP-GT scavenging was obtained as 32.07-91.034 and $46.75-96.12 \mu \mathrm{g} / \mathrm{ml}$, respectively.

The concept of $\mathrm{IC}_{50}$ was used in numerous studies to report the results of $\mathrm{DPPH} . \mathrm{IC}_{50}$ is defined as the concentration of GT or CS-NP-GT needed to inhibit $50 \%$ of DPPH radical. The less values of $\mathrm{IC}_{50}$ represent higher antioxidant power [29]. The $\mathrm{IC}_{50}$ of GT and CS-NP-GT were determined as $12.30 \pm 0.01$ and $6.13 \pm 0.12 \mu \mathrm{g} / \mathrm{ml}$, respectively, which indicated that the antioxidant activity of GT was high and increased by encapsulating (Figure 7). Ghahfarokhi et al. [30] reported a similar increase in the antioxidant power of thyme essential oil after encapsulating with CS so that free radical scavenging activity improved by $24.45 \%$ after entrapment in the CS-NP. Sessa et al. [31] reported the same results when measuring antioxidant activity of capsulated polyphenols from grape marc by oxygen radical absorbance capacity (ORAC) assay.

Ultrasonic treatment during producing CS-NP-GT is regarded as one of the reasons for increasing the antioxidant power of CS-NP-GT. Based on the previous studies, sonication can result in increasing DPPH free radical scavenging activity and antioxidant capacity. Regarding the study conducted on grape juice, its sonication treatment leads to an increase in its antioxidant capacity [32]. 


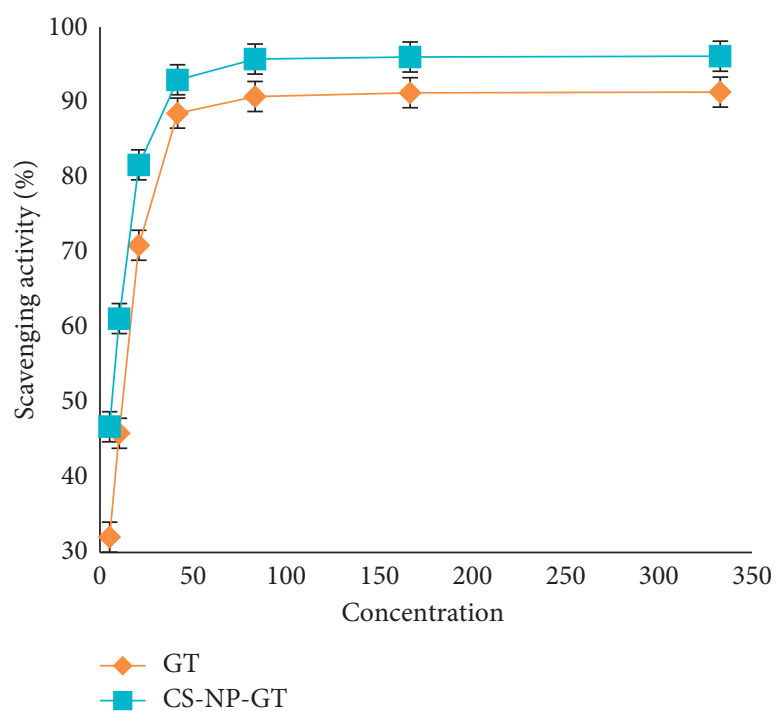

(a)

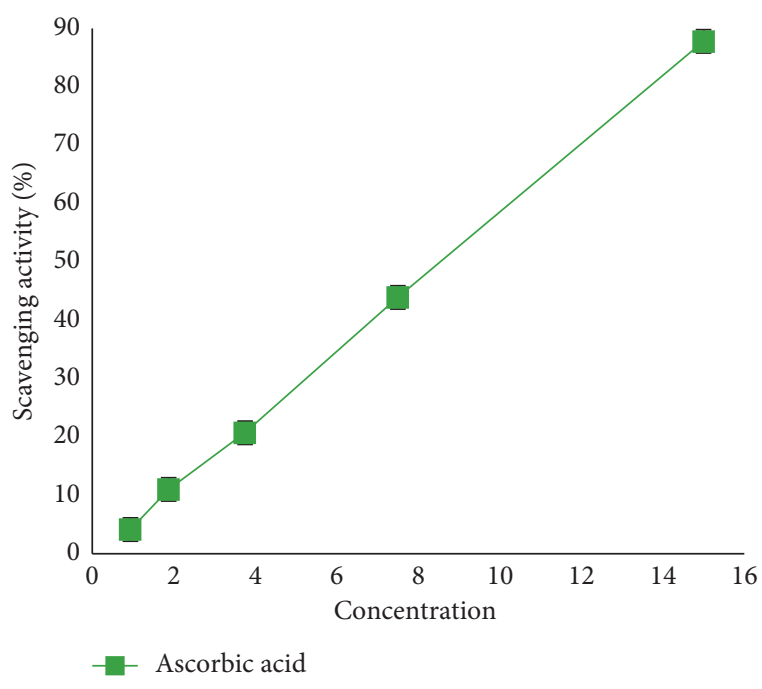

(b)

FIGURE 6: DPPH scavenging activity (\%) of GT and CS-NP-GT prepared using an initial weight ratio of CS : GT of $1: 0.5$ (a) and ascorbic acid (b).

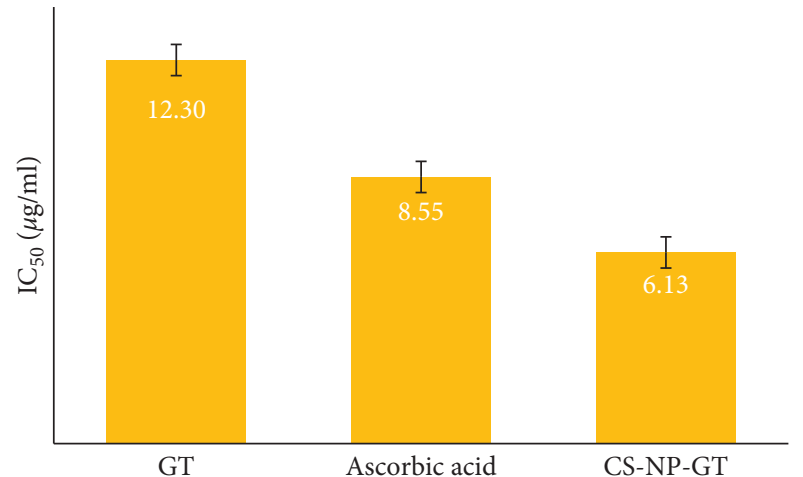

Figure 7: IC $_{50}$ of GT, CS-NP-GT (CS: GT =1:0.5), and ascorbic acid.

3.6. Total Phenolic Content. GT possesses high polyphenolic content and is considered as a good source of natural antioxidants. In general, extraction solvent, temperature, and solid-liquid ratio are considered as the factors affecting the process of extracting phenolic compounds from GT [33]. Water is regarded as a safe and environmentally friendly solvent. Further, maximum extraction yield and antioxidant activity were observed in the tea extracted by hot water [34]. Thus, water was used as the solvent for extracting GT extract. The TPC of GT and CS-NP-GT was determined by using Folin-Ciocalteu reagent based on gallic acid standard as $267.43 \pm 20.37$ and $302.19 \pm 2.67 \mathrm{mgr} / \mathrm{gr}$, respectively. Finally, TPC increased after encapsulating due to the addition of the hydroxyl groups produced in sonication step to the aromatic ring of phenolic compounds [32].

\section{Conclusions}

Although plants such as GT possess wonderful functional properties, applying these properties in foods necessitates the use of some methods such as nanoencapsulation for increasing the power of their active components and preventing organoleptic changes in foods. The results indicated that GT was successfully loaded in CS-NP. Based on the comparison of the particle size, zeta potential, PDI, and EE related to different CS : GT ratios, the amount of loaded GT affects the main properties of CS-NP-GT effectively. Measuring the antioxidant activity by DPPH method indicated that the scavenging activity of CS-NP-GT increases compared to that of free GT. Further, TPC enhances after encapsulating. Finally, this innovative method can increase the antioxidant activity of GT by considering the results of the present study.

\section{Data Availability}

The data used to support the findings of this study are available from the corresponding author upon request.

\section{Conflicts of Interest}

The authors declare that they have no conflicts of interest.

\section{References}

[1] A. V. S. Perumalla and N. S. Hettiarachchy, "Green tea and grape seed extracts-potential applications in food safety and quality," Food Research International, vol. 44, no. 4, pp. 827-839, 2011.

[2] R. Dominguez-Perles, D. A. Moreno, M. Carvajal, and C. Garcia-Viguera, "Composition and antioxidant capacity of a novel beverage produced with green tea and minimallyprocessed byproducts of broccoli," Innovative Food Science \& Emerging Technologies, vol. 12, no. 3, pp. 361-368, 2011.

[3] S. N. Senanayake, "Green tea extract: chemistry, antioxidant properties and food applications-a review," Journal of Functional Foods, vol. 5, no. 4, pp. 1529-1541, 2013. 
[4] D. Pasrija, P. N. Ezhilarasi, D. Indrani, and C. Anandharamakrishnan, "Microencapsulation of green tea polyphenols and its effect on incorporated bread quality," LWT-Food Science and Technology, vol. 64, no. 1, pp. 289296, 2015.

[5] A. F. M. Bora, S. Ma, X. Li, and L. Liu, "Application of microencapsulation for the safe delivery of green tea polyphenols in food systems: review and recent advances," Food Research International, vol. 105, pp. 241-249, 2018.

[6] J. Liang, F. Li, Y. Fang et al., "Synthesis, characterization and cytotoxicity studies of chitosan-coated tea polyphenols nanoparticles," Colloids and Surfaces B: Biointerfaces, vol. 82, no. 2, pp. 297-301, 2011.

[7] B. Jamil, R. Abbasi, S. Abbasi et al., "Encapsulation of cardamom essential oil in chitosan nano-composites: in-vitro efficacy on antibiotic-resistant bacterial pathogens and cytotoxicity studies," Frontiers in Microbiology, vol. 7, p. 1580, 2016.

[8] M. Beyki, S. Zhaveh, S. T. Khalili et al., "Encapsulation of Mentha piperita essential oils in chitosan-cinnamic acid nanogel with enhanced antimicrobial activity against Aspergillus flavus," Industrial Crops and Products, vol. 54, pp. 310-319, 2014.

[9] V. Darras, M. Nelea, F. M. Winnik, and M. D. Buschmann, "Chitosan modified with gadolinium diethylenetriaminepentaacetic acid for magnetic resonance imaging of DNA/chitosan nanoparticles," Carbohydrate Polymers, vol. 80, no. 4, pp. 1137-1146, 2010.

[10] Z. Rafiee, M. Barzegar, M. A. Sahari, and B. Maherani, "Nanoliposomal carriers for improvement the bioavailability of high-valued phenolic compounds of pistachio green hull extract," Food Chemistry, vol. 220, pp. 115-122, 2017.

[11] S. Woranuch and R. Yoksan, "Eugenol-loaded chitosan nanoparticles: I. Thermal stability improvement of eugenol through encapsulation," Carbohydrate Polymers, vol. 96, no. 2, pp. 578-585, 2013.

[12] A. J. Afolayan, F. O. Jimoh, M. O. Sofidiya, S. Koduru, and F. B. Lewu, "Medicinal potential of the root of Arctotis arctotoides," Pharmaceutical Biology, vol. 45, no. 6, pp. 486-493, 2007.

[13] T. Gutfinger, "Polyphenols in olive oils," Journal of the American Oil Chemists' Society, vol. 58, no. 11, pp. 966-968, 1981.

[14] N. Hasheminejad, F. Khodaiyan, and M. Safari, "Improving the antifungal activity of clove essential oil encapsulated by chitosan nanoparticles," Food Chemistry, vol. 275, pp. 113122, 2019.

[15] V. Arulmozhi, K. Pandian, and S. Mirunalini, "Ellagic acid encapsulated chitosan nanoparticles for drug delivery system in human oral cancer cell line (KB)," Colloids and Surfaces B: Biointerfaces, vol. 110, pp. 313-320, 2013.

[16] E. Tang, M. Huang, and L. Lim, "Ultrasonication of chitosan and chitosan nanoparticles," International Journal of Pharmaceutics, vol. 265, no. 1-2, pp. 103-114, 2003.

[17] M. Hasan, N. Belhaj, H. Benachour et al., "Liposome encapsulation of curcumin: physico-chemical characterizations and effects on MCF7 cancer cell proliferation," International Journal of Pharmaceutics, vol. 461, no. 1-2, pp. 519-528, 2014.

[18] M. Tsai, S. Bai, and R. Chen, "Cavitation effects versus stretch effects resulted in different size and polydispersity of ionotropic gelation chitosan-sodium tripolyphosphate nanoparticle," Carbohydrate Polymers, vol. 71, no. 3, pp. 448-457, 2008.
[19] A. G. Luque-Alcaraz, M. O. Cortez-Rocha, C. A. VelázquezContreras et al., "Enhanced antifungal effect of chitosan/ pepper tree (Schinus molle) essential oil bionanocomposites on the viability of Aspergillus parasiticus spores," Journal of Nanomaterials, vol. 2016, Article ID 6060137, 10 pages, 2016.

[20] D. L. Pavia, G. M. Lampman, G. S. Kriz, and J. A. Vyvyan, Introduction to Spectroscopy, Cengage Learning, Boston, MA, USA, 2008.

[21] J. Khouri, A. Penlidis, and C. Moresoli, "Viscoelastic properties of crosslinked chitosan films," Processes, vol. 7, no. 3, p. 157, 2019.

[22] U. Siripatrawan and B. R. Harte, "Physical properties and antioxidant activity of an active film from chitosan incorporated with green tea extract," Food Hydrocolloids, vol. 24, no. 8, pp. 770-775, 2010.

[23] M. Curcio, F. Puoci, F. Iemma et al., "Covalent insertion of antioxidant molecules on chitosan by a free radical grafting procedure," Journal of Agricultural and Food Chemistry, vol. 57, no. 13, pp. 5933-5938, 2009.

[24] C. Zhang, Y. Ding, Q. Ping, and L. Yu, "Novel chitosanderived nanomaterials and their micelle-forming properties," Journal of Agricultural and Food Chemistry, vol. 54, no. 22, pp. 8409-8416, 2006.

[25] B. W. S. Souza, M. A. Cerqueira, J. T. Martins, A. Casariego, J. A. Teixeira, and A. A. Vicente, "Influence of electric fields on the structure of chitosan edible coatings," Food Hydrocolloids, vol. 24, no. 4, pp. 330-335, 2010.

[26] J. Liang, H. Yan, X. Wang et al., "Encapsulation of epigallocatechin gallate in zein/chitosan nanoparticles for controlled applications in food systems," Food Chemistry, vol. 231, pp. 19-24, 2017.

[27] Q. Gan and T. Wang, "Chitosan nanoparticle as protein delivery carrier-systematic examination of fabrication conditions for efficient loading and release," Colloids and Surfaces B: Biointerfaces, vol. 59, no. 1, pp. 24-34, 2007.

[28] S. B. Kedare and R. P. Singh, "Genesis and development of DPPH method of antioxidant assay," Journal of Food Science and Technology, vol. 48, no. 4, pp. 412-422, 2011.

[29] O. P. Sharma and T. K. Bhat, "DPPH antioxidant assay revisited," Food Chemistry, vol. 113, no. 4, pp. 1202-1205, 2009.

[30] M. G. Ghahfarokhi, M. Barzegar, M. Sahari, and M. Azizi, "Enhancement of thermal stability and antioxidant activity of thyme essential oil by encapsulation in chitosan nanoparticles," Journal of Agricultural Science and Technology, vol. 18, pp. 1781-1792, 2016.

[31] M. Sessa, A. A. Casazza, P. Perego, R. Tsao, G. Ferrari, and F. Donsì, "Exploitation of polyphenolic extracts from grape marc as natural antioxidants by encapsulation in lipid-based nanodelivery systems," Food and Bioprocess Technology, vol. 6, no. 10, pp. 2609-2620, 2013.

[32] R. M. Aadil, X.-A. Zeng, Z. Han, and D.-W. Sun, "Effects of ultrasound treatments on quality of grapefruit juice," Food Chemistry, vol. 141, no. 3, pp. 3201-3206, 2013.

[33] D. Pasrija and C. Anandharamakrishnan, "Techniques for extraction of green tea polyphenols: a review," Food and Bioprocess Technology, vol. 8, no. 5, pp. 935-950, 2015.

[34] R. Farhoosh, G. A. Golmovahhed, and M. H. H. Khodaparast, "Antioxidant activity of various extracts of old tea leaves and black tea wastes (Camellia sinensis L.)," Food Chemistry, vol. 100, no. 1, pp. 231-236, 2007. 